\title{
Generative Innovation Practices, Customer Creativity, and the Adoption of New Technology Products
}

\author{
Stoyan Tanev and Marianne Harbo Frederiksen
}

\author{
"(It is absurd to claim that our customers are missing!") \\ So say surprised skeptics seeing our claim of missing \\ customers. What if our problem with value is rooted \\ in a misconception of our customers, the people we \\ are creating value for?
}

Peter J. Denning \& Robert P. Dunham "The Missing Customer" (2003; tinyurl.com/kl7y2wp)

\begin{abstract}
We offer a critical reflection on one of the key reasons for the startlingly low success rate of innovation initiatives worldwide - the fact that the interactive environment surrounding the customer is a critical part of the adoption process; it can and should be designed in a way that enables customer creativity, and thus adoption. In this article, we embrace a definition of innovation as "the adoption of a new practice by a community" where the innovator is the one who does not only sense and move into new opportunities but also mobilizes all the necessary resources needed by customers to adopt a new practice. The emphasis on adoption merges together innovation and entrepreneurship by shifting the focus from the inventor and the designer, through the entrepreneur, to the ultimate recipient of the innovative outcomes. Looking at customers as co-creators is critically important for technological product adoption; missing the chance to enable their creativity is equivalent to missing the opportunity of seeing them for who they really are. The result is a distorted vision that is ultimately rooted in the misconception of the dynamics of customer value. We particularly emphasize two points: i) the increasing degree of complexity of everyday technological products requires a higher degree of creativity by customers to adopt; and ii) customer creativity is not only a function of user-technology interaction, it is a function of the various actors in the interactive environment surrounding the customer such as other customers, other technologies, local distributors, customer/technical support providers, and competitors.
\end{abstract}

\section{Introduction}

According to a 2005 Business Week article, the success rate of innovation initiatives in terms of meeting their financial objectives is less than $4 \%$, with the innovation success rates within specific industries ranging from a mere $1 \%$ in the toy industry to only $7.5 \%$ in the pharmaceutical industry (Nussbaum, 2005; tinyurl.com/krb6oyv). In a more recent study, Strategyn (2010; tinyurl.com/ olgqvtp) used 12 different sources to evaluate the success rate of traditional innovation methods. The study re- ports success rates between $1 \%$ and $86 \%$, with an average success rate of $17 \%$. After removing the low and high outliers from the analysis, the average rate goes down to $8.5 \%$ - exactly half of the initially reported $17 \%$. A most recent study by Accenture (2013; tinyurl.com/ n7hdyb4) found that $93 \%$ of executives regard their company's long-term success to be dependent on its ability to innovate; but, at the same time, less than one out of five (18\%) believe that their strategic investments in innovation are paying off. According to the study, such a poor track record discourages companies from taking 


\section{Generative Innovation Practices, Customer Creativity, and Adoption}

Stoyan Tanev and Marianne Harbo Frederiksen

the risk of initiating more radical innovation projects. There is no doubt that the specific success rates reported by the different studies depend on the methodology, the purpose of the study, and the particular context of their key messages. However, they seem to consistently indicate that, at the beginning of the $21 \mathrm{st}$ century, human involvement in dedicated innovation activities has not been as successful as we have been expecting it to be. Many companies are simply struggling with it - a fact that has been borne out in numerous other studies as well as in the marketplace, where new product introductions quite often fail to meet expectations even as others soar beyond expectations. What is the reason for such discouraging performance? Should we just lower our expectations by admitting that innovation is a risky game and silently agree to waste more than $80 \%$ of our investments? Or, should we try to locate the roots of the cause and work towards improving the success rate? What can innovators and entrepreneurs do to improve it?

In this article, we argue that one of the reasons for such failure could be associated with narrow or fluffy definitions of innovation that are impossible to translate into actionable insights. The problem with inadequate definitions is that: i) they misinterpret the job of the innovator and the entrepreneur; and ii) they misplace the focus of company efforts into activities that do not enable potential customers to become actual customers thus making the companies "miss the customer." We start by considering innovation as "the adoption of a new practice by a community", which emphasizes the critical roles of both innovators/entrepreneurs and customers as the two active poles of the dynamic adoption process. The entrepreneurial aspects are addressed by describing a generative approach to managing innovation, including several personal practices focusing on adoption. The customer aspects are addressed by conceptualizing customer creativity as an important factor in the adoption process. The article concludes by emphasizing the relevance of the topic with respect to the ever-increasing complexity of everyday technological products and summarizing the key insights of the analysis.

\section{Innovation as the Adoption of a New Practice by a Community}

The particular working definition of innovation appears to be of critical importance for companies. Baregheh, Rowley, and Sambrook (2009; tinyurl.com/ko9r7h4) emphasize the fundamental difficulties in defining innovation by referring to its multidisciplinary nature. They have analyzed 60 definitions from eight fields including: business and management; economics; organization studies; innovation and entrepreneurship; technology, science and engineering; knowledge management; and marketing. Building on these diverse definitions, they propose a general and integrative definition that could be applied to the majority of contexts: "Innovation is the multi-stage process whereby organizations transform ideas into new/improved products, services, or processes, in order to advance, compete, and differentiate themselves successfully in their marketplace."

In this article, we embrace a definition suggested by Denning and Dunham (2010; innovators-way.com) who stress that successful innovation cannot be completed until the community of the intended users has actually adopted a new practice. For them, innovation is "the adoption of a new practice by a community". With such a definition, the focus of innovation shifts from invention to adoption practices and emphasizes the fact that there are millions of inventions that have never found their way to the marketplace. Interestingly, Accenture's (2013; tinyurl.com/n7hdyb4) study mentioned earlier found that one of the key reasons for the low efficiency of companies' innovation activities is the so-called "invention trap" - the "overreliance on the invention process itself to produce success and relative lack of systematic, enterprise-wide processes capable of commercializing inventions into products or services at scale, bringing them to market in a sufficiently timely fashion and reaping the expected returns."

The key benefit of the definition provided by Denning and Dunham is that it decouples the practices of invention from the practice of innovation which focuses on enabling adoption. This decoupling has two main effects. First, it merges together innovation and entrepreneurship, because they both could now be considered as managing and implementing change as part of the adoption of new practices. Second, it opens the opportunity to account for the value co-creation role of customers during the adoption process - a point that needs to be strongly emphasized. The two effects should be considered in a self-consistent manner because they are dialectally interrelated.

\section{A Generative Approach to Managing Innovation as Adoption}

Denning and Dunham (2010; innovators-way.com) have developed a generative approach to managing innovation, which consists of eight practices within three 


\section{Generative Innovation Practices, Customer Creativity, and Adoption}

\section{Stoyan Tanev and Marianne Harbo Frederiksen}

categories: i) the work of invention, including the practices of sensing and envisioning; ii) the work of adoption, including the practices of offering, adopting, and sustaining; and iii) the three practices providing the environment for all the other practices, including executing, leading, and embodying. One of the key messages of this classification is that the major work of innovation is not related to invention but rather to the personal practices of innovators and entrepreneurs aiming at getting others to adopt a new practice enabled by a new product, process, or service. Offering is the first such practice including the presentation of a proposed new practice and its benefits to the community and its leaders so that they commit to considering it. Adopting is getting the community members to commit to adopting the practice for the first time, while reserving the option of dropping it if not satisfied after a trial period. Sustaining consists of getting the community members to commit to the practice for an extended period, integrating it into their other practices, standards, incentives, and processes, and making it productive for its useful life.

Denning and Dunham (2010; innovators-way.com) identify the following key activities associated with the offering practice:

- drawing listeners into a discussion about the ways of producing the new outcome

- modifying the proposal to fit listeners' concerns

- establishing trust in your expertise to fulfill the offer

They identify the following key activities associated with the adopting practice:

- achieving initial commitment to the new practice

- continuously demonstrating the value of the new practice

- showing how to manage risks and deal with resistance

- aligning action plans for coherence with existing practices, concerns, and interests

- addressing different community member adoption rates

- recruiting allies
- developing marketing strategies for the different groups in the community

- continuously look for ways to overcome resistance

And finally, they identify the following key activities associated with the sustaining practice:

- achieving commitment to stick with new practice

- developing supporting mechanisms, tools, and infrastructure

- integrating the new practice with the surrounding environment, standards, and incentive systems

- continuously assessing for negative consequences

- carefully abandoning bad or obsolete innovations

Denning and Dunham point out that the key activities associated with the three adoption practices should be considered at the personal level as conversational or rather discursive expressions of human behaviour. According to such a discursive perspective, the personality of the innovator or the entrepreneur should be considered in terms of the specific personal practices and their outcomes - "the streams of human actions and interactions, which can be understood in terms of their meanings for the actors and interactors and the norms and the traditions that are generally accepted by the people involved and which shape their actions" (Harré and Moghaddam, 2012; tinyurl.com/mq42vad).

It is true that conversation is very useful, but it is not the only model for analyzing such streams of action. However, it allows for treating all that people do collectively and individually, as well as privately and publicly, as if it were a kind of conversation or discourse - in other words, as consisting of meaningful exchanges constrained by a specific normative framework (Harré and Moghaddam, 2012; tinyurl.com/mq42vad). The entrepreneurial discursive skills and dispositions are a subset of human personal knowledge that most people possess to a certain extent but might not have been able to express, grow, or master. This realization has great implications for the study of entrepreneurship and innovation because it points out that the role of the learning process is to help all interested in entrepreneurship to discover the depths of their entrepreneurial self and nurture it in a consistent way. 


\section{Generative Innovation Practices, Customer Creativity, and Adoption}

\section{Customer Creativity as a Key Factor in Technology Adoption}

Denning and Dunham's approach has a great value in articulating the job of both innovators and entrepreneurs in terms of the specific practices that could be learned and perfected. Their approach, however, does not seem to sufficiently emphasize another important aspect - the fact that customers' activities are an equally important component of the adoption equation. We believe that the second major reason for the failure of the majority of innovation initiatives in the technology domain is the lack of proper understanding of the creativity needed by the ultimate users who are struggling to adopt the newly developed products. Our emphasis on customer creativity in the adoption of new products does not intend to undermine the efforts of designers, innovators, or entrepreneurs; it is just an attempt to locate another major source of the problem and suggest a way out of it. The solution includes the repositioning of the creativity concept within the context of customers' adoption efforts.

The widely acknowledged definition of creativity refers to the novelty, usefulness, and appropriateness of a new product (Duxbury, 2012; timreview.ca/article/594). However, this definition misses the important element of appropriation, which can be seen as a result of the creative efforts of the ultimate recipients of the new product. The increasing complexity of new technological products enlarges the difference between the total value built in as part of the design, development, and manufacturing process and the customer's perspective of that value. The difference allows us to emphasize two points. First, potential customers make purchase and adoption decisions on the basis of the relative benefit 1 , which is the difference between the total value (reflecting the entrepreneurial perspective) and the value of whatever their currently existing solution is (Figure 1). Second, the estimation of the relative benefit 1 is based on the assumption that customers know in advance what the total value of a product is. It assumes that the total value is an objectively existing property that could be easily appreciated by potential customers. This last assumption is not true, especially in the case of more complex technology-based products. What customers really know is the perceived value of the product and, unfortunately, this perceived value could be lower than the value of their existing solution, leading to a negative relative benefit 2 . In such situations, customers have two options: either neglect the new product or make the effort to further appreciate the total value of the new product.

\section{Entrepreneurial Perspective}

\section{Customer Perspective}

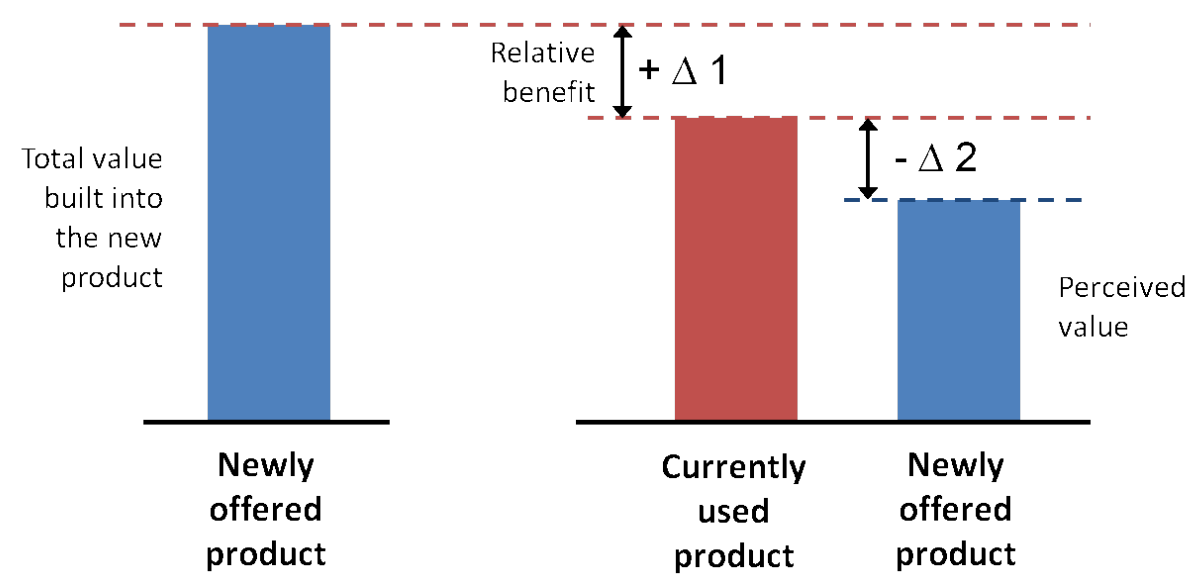

Figure 1. Visualization of the difference between the total value of a new product and its perceived customer value. Modified from Adner (2012; thewidelensbook.com). 


\section{Generative Innovation Practices, Customer Creativity, and Adoption}

Stoyan Tanev and Marianne Harbo Frederiksen

The reason for us to focus in greater detail on the difference between the total and the perceived value of a new product is to emphasize that: i) an adoption decision does not happen before there is a positive difference between the perceived value of a newly offered product and the value of the existing solution used by the potential adopters of the new product, and ii) this process takes time and effort on the side of the potential customers. In this sense, the perception that will make a specific potential customer buy and adopt is to a great extent the result of this customer's own activities and creative efforts - in other words, it should be conceptualized as customer creativity.

Product attributes are manifested within the context of specific circumstances. For example, two different customers may associate an original technological product with completely different perceptions depending on the degree of their actual involvement and creative efforts in actively appreciating its use value. One could actually speak of this association as a process of "product co-creation" given that the evolution of the perception of a particular product makes sense only within the specific context of a particular customer. In other words, every customer co-creates the product for him or herself using accessible resources. In this sense, customer creativity is always co-creativity; it is dialogical and relational. The dialogue and the relations go far beyond the activities emerging within the context of the dyad formed by the user and the technology to include all possible insights from a variety of actors in the interactive environment surrounding the customer, such as other customers, other technologies, local distributors, customer/technical support providers, and competitors. This realization suggests that activity-based approaches such as actor-network theory (Latour, 2005; tinyurl.com/m99un78) and activity theory (Kaptelinin and Nardi, 2006; tinyurl.com/m4qp8s3) could be highly appropriate in studying the dynamics and the outcomes of product adoption.

\section{The Increasing Complexity of Everyday Technological Products}

The discussion of customer creativity suggested here is justified by the realization that there is an increased degree of complexity in most of the technological products used in everyday human lives. The higher degree of complexity generates both societal and personal pressures that are in the process of changing many aspects of the human condition. Scale is one of the critical concepts that could help in understanding how societal pressures are resulting in a significantly increased degree of technological complexity. It refers to the unprecedented increase of human population, the increasing intensity of the globalization processes, and the increasing relevance of technology in everyday human life. The increasing scale of society is forcing a shift from trust and trustworthiness based on personal relationships to impersonal trust, predictability, and compliance in both people and systems, which leads to different societal pressures from a number of different directions (Schneider, 2012; tinyurl.com/mcj8xwf):

1. Having more people in society changes the effectiveness of different reputational pressures driven by the necessity for the majority of people to follow dominant group norms due to fear from bad reputation.

2. There is a visible tendency for an increased degree of complexity of everyday technological products, given that having more people in society means more interactions among people. More interactions among people cause both the emergence of new societal dilemmas and interdependencies among them. The interdependency of newly emerging dilemmas requires new and more complex social management systems that need to rely on technology even more. Uncertainty is a key component of new technology development and more technology means that the new systems may have more flaws as well as a higher risk of failing in surprising and unexpected ways, which additionally complicates the entire socio-technological environment.

3. There is a growing variety of new technological systems. As more and different technology permeates human lives and society in general, there will be new areas of concern that will need to be addressed, new societal dilemmas, and newly emerging technological challenges. In this context, the concept of scale in society becomes even more important because more aspects of our society are going to be controlled not by people but by technologically automated systems. Unfortunately, the ongoing automation of social systems is paralleled by a process of depersonalization of the interaction between people, which additionally increases social pressures due to the inability to efficiently clarify problems associated with communication ambiguities.

4. Globalization has brought the opportunity for people to move much greater distances across national borders, across nations, and across continents. Greater 


\title{
Generative Innovation Practices, Customer Creativity, and Adoption
}

\author{
Stoyan Tanev and Marianne Harbo Frederiksen
}

distances create the potential for more people, with weaker social ties, to be involved in mutual accidental interactions, which may weaken their moral and reputational pressures and diminish the strength of their home-based institutional pressures. This situation creates a necessity for more control and more monitoring, not only of people, but also of unprecedented amounts of goods and services, which additionally enhances the need for more complex technological solutions based on wireless, sensing, information and communication technologies.

\section{Conclusion}

In this article, we embraced a definition of innovation as "the adoption of a new practice by a community" where the innovator is the one who mobilizes all the necessary resources to enable customers to adopt the new practice. One of the benefits of such a definition is that it merges together innovation and entrepreneurship and shifts the focus from the inventor and the designer to the entrepreneur and the ultimate adopters of the innovative outcomes. The entrepreneurial aspects of technology adoption were discussed by summarizing the generative practices adoption framework suggested by Denning and Dunham (2010; innovators-way.com). We have, however, also emphasized the relevance of customers' creative efforts and activities as a key factor in the adoption process and suggested conceptualizing these efforts as part of customer creativity. The point of this emphasis is to underline the fact that customer creativity is another key prerequisite for the success of innovation initiatives. Failing to integrate the mastership of the personal innovation practices to the design and development of a commercialization environment that enables the co-creativity of customers will always result in missing the customers as the ultimate destination of the firm's offerings.

\section{About the Authors}

Stoyan Tanev is an Associate Professor in the Department of Technology and Innovation and member of the Centre for Integrative Innovation Management at the University of Southern Denmark, Odense, Denmark, as well as Adjunct Professor in the Department of Systems and Computer Engineering at Carleton University in Ottawa, Canada, where he was previously a faculty member in the Technology Innovation Management Program. He has a MSc and a PhD in Physics jointly from the University Pierre and Marie Curie, Paris, France and the University of Sofia, Bulgaria, a PhD in Theology from the University of Sofia, Bulgaria, an MEng in Technology Management from Carleton University, Canada, and an MA from the University of Sherbrooke, Canada. He has multidisciplinary research interests with a focus on the fields of technology innovation management, born global technology startup business model development and value co-creation. Dr. Tanev is Senior IEEE member and member of the Review Board of the Technology Innovation Management Review.

Marianne Harbo Frederiksen is an Associate Professor in the Department of Technology and Innovation and a member of the Centre for Integrative Innovation Management at the University of Southern Denmark. Currently, she is also a PhD student focusing on creative processes and outcomes in connection with new product development and adoption and therefore the linkages between creativity and innovation. She has an MSc in Architecture from the Aarhus School of Architecture, Denmark, with a specialization within industrial design and product development. She has been co-owner of a design company and has worked in and together with several industries as a designer and R\&D Manager as well as an adviser in public-private research projects focusing on user experience, experience designing, and other aspects of product development.
Citation: Tanev, S. and M.H. Frederiksen. 2014. Generative Innovation Practices, Customer Creativity, and the Adoption of New Technology Products. Technology Innovation Management Review. February 2014: 5-10.

Keywords: entrepreneurship, innovation, technology adoption, customer creativity, co-creation, customer value 